\title{
Effective High-Order Iterative Methods via the Asymptotic Form of the Taylor-Lagrange Remainder
}

\begin{abstract}
Isaac Fried
Department of Mathematics, Boston University, Boston, MA 02215, USA

Correspondence should be addressed to Isaac Fried; if@math.bu.edu

Received 18 April 2014; Revised 27 May 2014; Accepted 29 May 2014; Published 3 July 2014

Academic Editor: Filomena Cianciaruso

Copyright ( 2014 Isaac Fried. This is an open access article distributed under the Creative Commons Attribution License, which permits unrestricted use, distribution, and reproduction in any medium, provided the original work is properly cited.

The asymptotic form of the Taylor-Lagrange remainder is used to derive some new, efficient, high-order methods to iteratively locate the root, simple or multiple, of a nonlinear function. Also derived are superquadratic methods that converge contrarily and superlinear and supercubic methods that converge alternatingly, enabling us not only to approach, but also to bracket the root.
\end{abstract}

\section{The Asymptotic Form of the Taylor-Lagrange Remainder}

The Taylor-Lagrange theorem is a corollary of extended Rolle's theorem, which for the spare polynomial function

$$
f(x)=x^{n}(1-x), \quad 0 \leq x \leq 1
$$

such that

$$
\begin{gathered}
f(0)=f^{\prime}(0)=f^{\prime \prime}(0)=\cdots=f^{(n-1)}(0)=0, \\
f^{(n)}(0) \neq 0, \quad f(1)=0
\end{gathered}
$$

is exactly

$$
f^{(n)}(\xi)=0, \quad 0<\xi=\frac{1}{n+1}<1 .
$$

As $n$, the degree of osculation at point $x=0$, increases, $\xi$ inexorably edges leftwards toward that point.

The Taylor-Lagrange theorem states that if function $f(t)$ is continuous in the closed interval $[a, x]$ and is $n$ times differentiable in the open interval $(a, x)$ and $f^{(n-1)}(a)$ exists, then

$$
f(x)=p_{n-1}(x)+\frac{1}{n !}(x-a)^{n} f^{(n)}(\xi), \quad a<\xi<x
$$

with the osculating polynomial part of the formula being

$$
\begin{aligned}
p_{n-1}(x)= & f(a)+f^{\prime}(a)(x-a)+\frac{1}{2 !} f^{\prime \prime}(a)(x-a)^{2}+\cdots \\
& +\frac{1}{(n-1) !} f^{(n-1)}(a)(x-a)^{n-1} .
\end{aligned}
$$

As with extended Rolle's theorem, as $n$ increases, $\xi$ moves gradually closer to point $a$. More precisely,

Theorem 1. If in addition to the required conditions on the function in the Taylor-Lagrange formula, also $f^{(n+1)}(a) \neq 0$ and $f^{(n+1)}(t)$ exists in $[a, x]$ and is continuous from the right at $t=a$, then $\xi$ in (4) is such that

$$
\lim _{x \rightarrow a^{+}} \frac{\xi-a}{x-a}=\frac{1}{n+1}
$$

or

$$
\xi=\frac{n}{n+1} a+\frac{n}{n+1} x
$$

nearly, if $x$ is nearly a, implying that

$$
\begin{aligned}
f(x)= & p_{n-1}(x)+\frac{1}{n !}(x-a)^{n} f^{(n)}\left(\frac{n}{n+1} a+\frac{n}{n+1} x\right) \\
& +R(x)
\end{aligned}
$$


with

$$
R(x)=O\left((x-a)^{k}\right), \quad k \geq n+2 .
$$

We will give here an explicit elementary proof to this theorem for $n=2$ only; for its generalization, see $[1,2]$.

We write the Taylor-Lagrange formula for $n=2, a=0$

$$
f(x)=f(0)+x f^{\prime}(0)+\frac{1}{2} x^{2} f^{\prime \prime}(\xi), \quad 0<\xi<x
$$

so as to have, with $\xi=k x$,

$$
f(x)=f(0)+x f^{\prime}(0)+\frac{1}{2} x^{2} f^{\prime \prime}(k x)+R(x)
$$

and seek $k$ so that $R(x)=O\left(x^{4}\right)$.

Using L'Hopital's rule, we determine that

$$
\lim _{x \rightarrow 0} \frac{R(x)}{x^{3}}=\frac{1}{6}(1-3 k) f^{\prime \prime \prime}(0) .
$$

We take $k=1 / 3=1 /(n+1)$ and establish that the asymptotic error with this $k$ is

$$
R(x \sim 0)=\frac{1}{72} x^{4} f^{\prime \prime \prime \prime}(0) .
$$

For example

$$
\begin{gathered}
\ln (1+x)=x-\frac{9}{2}\left(\frac{x}{3+x}\right)^{2}+R(x), \\
R(x \sim 0)=-\frac{1}{12} x^{4} .
\end{gathered}
$$

\section{From Newton to Halley via the Asymptotic Remainder}

We write the $n=1$ version of the Taylor-Lagrange formula

$$
\begin{aligned}
f(x)=f\left(x_{0}+\delta x\right)= & f\left(x_{0}\right)+\delta x f^{\prime}(\xi), \\
& x_{0}<\xi<x_{0}+\delta x
\end{aligned}
$$

and obtain from it, by setting $f(x)=0, \xi=x_{0}$, the classical Newton method

$$
\begin{aligned}
& x_{1}=x_{0}+\delta x=x_{0}-u_{0}, \quad \delta x=-u_{0}, \\
& u_{0}=\frac{f_{0}}{f_{0}^{\prime}}, \quad f_{0}=f\left(x_{0}\right), \quad f_{0}^{\prime}=f^{\prime}\left(x_{0}\right)
\end{aligned}
$$

which is, as is well known, a second order method

$$
x_{1}-a=\frac{1}{2} \frac{f^{\prime \prime}}{f^{\prime}}\left(x_{0}-a\right)^{2}+O\left(\left(x_{0}-a\right)^{3}\right),
$$

provided that $f^{\prime}=f^{\prime}(a) \neq 0$. In the above equation $x_{0}$ is the input and $x_{1}$ is the output of the iterative process.

The difficult problem of finding the root of the nonlinear function $f(x)$ is replaced in Newton's method by the easy task of repeatedly finding the approximating root of the tangent line $g(x)=f_{0}^{\prime}\left(x-x_{0}\right)+f_{0}$. This is the essence of all other higher-order methods, to supplant the finding of the root of the original function by the repeated finding of the root of an approximating polynomial.

Having computed $x_{1}$ by (16), it occurs to us to return and replace the initial $\xi=x_{0}$ by the asymptotic

$$
\xi=\frac{x_{0}+x_{1}}{2}=x_{0}+\frac{1}{2} \delta x, \quad \delta x=-u_{0}=-\frac{f_{0}}{f_{0}^{\prime}}
$$

to have the two-step, mid-point method

$$
x_{2}=x_{0}-\frac{f\left(x_{0}\right)}{f^{\prime}\left(x_{0}+(1 / 2) \delta x\right)}, \quad \delta x=-u_{0}=-\frac{f_{0}}{f_{0}^{\prime}}
$$

which is cubic or third order

$$
x_{2}-a=\frac{1}{24} \frac{6 f^{\prime \prime 2}-f^{\prime} f^{\prime \prime \prime}}{f^{\prime 2}}\left(x_{0}-a\right)^{3}+O\left(\left(x_{0}-a\right)^{4}\right) \text {. }
$$

See also Traub [3, page 164, (8)-(12)].

The linearization

$$
\begin{gathered}
f^{\prime}\left(x_{0}+\frac{1}{2} \delta x\right)=f^{\prime}\left(x_{0}\right)+\frac{1}{2} \delta x f^{\prime \prime}\left(x_{0}\right), \\
\delta x=-u_{0}=-\frac{f\left(x_{0}\right)}{f^{\prime}\left(x_{0}\right)}=-\frac{f_{0}}{f_{0}^{\prime}}
\end{gathered}
$$

reproduces out of (19) classical Halley's method

$$
\begin{aligned}
x_{1} & =x_{0}+\frac{\operatorname{det}\left[\begin{array}{cc}
-1 & f_{0} \\
0 & 2 f_{0}^{\prime}
\end{array}\right]}{\operatorname{det}\left[\begin{array}{cc}
f_{0}^{\prime} & f_{0} \\
f_{0}^{\prime \prime} & 2 f_{0}^{\prime}
\end{array}\right]} f_{0}=x_{0}-\frac{2 f_{0}^{\prime}}{2 f_{0}^{\prime 2}-f_{0} f_{0}^{\prime \prime}} f_{0} \\
& =x_{0}-\frac{1}{1-\left(f_{0}^{\prime \prime} / 2 f_{0}^{\prime}\right) u_{0}} u_{0},
\end{aligned}
$$

which is cubic as well,

$$
x_{1}-a=\frac{1}{12} \frac{3 f^{\prime \prime 2}-2 f^{\prime} f^{\prime \prime \prime}}{f^{\prime 2}}\left(x_{0}-a\right)^{3}+O\left(\left(x_{0}-a\right)^{4}\right) \text {, }
$$

but requires the second derivative $f^{\prime \prime}(x)$.

Power series expansion modifies rational Halley's method into the polynomial in $u_{0}$ form

$$
x_{1}=x_{0}-u_{0}-\frac{1}{2} \frac{f_{0}^{\prime \prime}}{f_{0}^{\prime}} u_{0}^{2}, \quad u_{0}=\frac{f_{0}}{f_{0}^{\prime}},
$$

which is still cubic,

$$
x_{1}-a=\frac{1}{6} \frac{3 f^{\prime \prime 2}-f^{\prime} f^{\prime \prime \prime}}{f^{\prime 2}}\left(x_{0}-a\right)^{3}+O\left(\left(x_{0}-a\right)^{4}\right)
$$

provided that $f^{\prime}=f^{\prime}(a) \neq 0$. See also Traub [3, page 205, (9)-(44)]. 
We write the equation of the osculating parabola

$$
g(x)=f_{0}+f_{0}^{\prime}\left(x-x_{0}\right)+\frac{1}{2} f_{0}^{\prime \prime}\left(x-x_{0}\right)^{2}
$$

to $f(x)$ at $x_{0}$ and seek its intersection with the $x$-axis. The smaller root, $x_{1}$, of $g(x)$ is given by

$$
\begin{array}{r}
x_{1}-x_{0}=\frac{-f_{0}^{\prime}+\sqrt{f_{0}^{\prime 2}-2 f_{0}^{\prime \prime} f_{0}}}{f_{0}^{\prime \prime}}=-u_{0}-\frac{1}{2} \frac{f_{0}^{\prime \prime}}{f_{0}^{\prime}} u_{0}^{2}+O\left(u_{0}^{3}\right), \\
u_{0}=\frac{f_{0}}{f_{0}^{\prime}}
\end{array}
$$

which is Halley's method of (24).

\section{Construction of High-Order Iterations by Generalized Undetermined Coefficients}

Halley's method or, for that matter, any other higher-order method such as that in (24) can be derived ab initio by writing $\delta x, x_{1}=x_{0}+\delta x$ as a power series of $u_{0}=f_{0} / f_{0}^{\prime}$ or merely $f_{0}=f\left(x_{0}\right)$, as in

$$
x_{1}=x_{0}+P f_{0}+Q f_{0}^{2},
$$

and then progressively fixing the undetermined coefficients $P$ and $Q$, which eventually need not remain constant, so as to achieve the highest possible order of convergence.

Thus, at first, we have from (28) that

$$
x_{1}-a=\left(1+P f^{\prime}\right)\left(x_{0}-a\right)+O\left(\left(x_{0}-a\right)^{2}\right) \text {. }
$$

We substitute variable $f^{\prime}\left(x_{0}\right)$ for the constant $f^{\prime}(a)$ in (29) and try $P=-1 / f_{0}^{\prime}$. With this $P$ we have next that

$$
x_{1}-a=\frac{2 Q f^{\prime 3}+f^{\prime \prime}}{2 f^{\prime}}\left(x_{0}-a\right)^{2}+O\left(\left(x_{0}-a\right)^{3}\right)
$$

and we set

$$
P=-\frac{1}{f_{0}^{\prime}}, \quad Q=-\frac{f_{0}^{\prime \prime}}{2 f_{0}^{\prime 3}}
$$

with which the polynomial variant of Halley's method in (24) is regained.

Doing the same to the rational method

$$
x_{1}=x_{0}+\frac{P+Q f_{0}}{R+S f_{0}} f_{0} \text {, }
$$

we verify that cubic convergence is achieved with $P=-1 / f_{0}^{\prime}$, $Q=0, R=1$, and $S=-f_{0}^{\prime \prime} /\left(2 f_{0}^{\prime 2}\right)$ as in classical Halley's method in (22).

For other interesting applications of the method of undetermined coefficients, see $[4,5]$.

\section{High-Order Iterative Methods Derived from the Weighted Fixed Point Iteration}

Consider the fixed point iteration

$$
x_{1}=F\left(x_{0}\right)
$$

for point $a, F(a)=a$. We write $x_{1}-a=F\left(x_{0}\right)-a$ and have the power series expansion

$$
\begin{aligned}
x_{1}-a= & F^{\prime}(a)\left(x_{0}-a\right)+\frac{1}{2 !} F^{\prime \prime}(a)\left(x_{0}-a\right)^{2} \\
& +\frac{1}{3 !} F^{\prime \prime \prime}(a)\left(x_{0}-a\right)^{3}+\cdots .
\end{aligned}
$$

Hence, if $\left|F^{\prime}(x)\right|<1$ around $x=a$, then the fixed point iteration converges linearly; and if $F^{\prime}(a)=0$, then the fixed point iteration converges quadratically, and so on.

Suppose now that we are seeking single root $a$ of $f(x), f(a)=0, f^{\prime}(a) \neq 0$. We rewrite $f(x)=0$ as the equivalent fixed point problem

$$
x=F(x), \quad F(x)=x+w(x) f(x)
$$

for weight function $w(x), w(a) \neq 0$, and seek to fix it to our advantage. For a quadratic method, $w(x)$ needs to be such that

$$
F^{\prime}(x)=1+w^{\prime}(x) f(x)+w(x) f^{\prime}(x)=0
$$

for $x$ near $a$. Since $f(a)=0$, we choose to ignore $w^{\prime}(x) f(x)$ in the previous equation and are left with $w\left(x_{0}\right)=-1 / f^{\prime}\left(x_{0}\right)$ and Newton's method.

From

$$
\begin{gathered}
F^{\prime}(x)=1+f^{\prime}(x) w(x)+f(x) w^{\prime}(x)=0, \\
F^{\prime \prime}(x)=f^{\prime \prime}(x) w(x)+2 f^{\prime}(x) w^{\prime}(x)+f(x) w^{\prime \prime}(x)=0
\end{gathered}
$$

and ignoring $f(x) w^{\prime \prime}(x)$ in the second equation, we obtain the system

$$
\left[\begin{array}{cc}
f^{\prime} & f \\
f^{\prime \prime} & 2 f^{\prime}
\end{array}\right]\left[\begin{array}{c}
w \\
w^{\prime}
\end{array}\right]=\left[\begin{array}{c}
-1 \\
0
\end{array}\right]
$$

which we solve for $w(x)$ as

$$
w\left(x_{0}\right)=\frac{\operatorname{det}\left[\begin{array}{cc}
-1 & f_{0} \\
0 & 2 f_{0}^{\prime}
\end{array}\right]}{\operatorname{det}\left[\begin{array}{cc}
f_{0}^{\prime} & f_{0} \\
f_{0}^{\prime \prime} & 2 f_{0}^{\prime}
\end{array}\right]}
$$

and arrive at Halley's method of (22). Higher-order methods are systematically generated in similar fashion. See also [6].

\section{The Recursive Generation of the High-Order Iteration Function}

Let $F_{2}(x)$

$$
F_{2}(a)=a, \quad F_{2}^{\prime}(a)=0
$$


be the fixed point iteration function of the recursion $x_{1}=$ $F_{2}\left(x_{0}\right)$. By dint of $F_{2}^{\prime}(a)=0$, the iterative method is quadratic

$$
x_{1}-a=\frac{1}{2} F_{2}^{\prime \prime}(a)\left(x_{0}-a\right)^{2}+O\left(\left(x_{0}-a\right)^{3}\right) \text {. }
$$

The recursively constructed iteration function

$$
F_{n+1}(x)=F_{n}(x)+\frac{1}{n} F_{n}^{\prime}(x)\left(F_{n}(x)-x\right), \quad n=2
$$

assures a third order convergence

$$
x_{1}-a=\frac{1}{12}\left(3 F_{2}^{\prime \prime}(a)^{2}-F_{2}^{\prime \prime \prime}(a)\right)\left(x_{0}-a\right)^{3}+O\left(\left(x_{0}-a\right)^{4}\right) .
$$

For example, taking

$$
F_{2}(x)=x-m u(x), \quad u(x)=\frac{f(x)}{f^{\prime}(x)},
$$

we obtain

$$
\begin{array}{r}
F_{3}(x)=x-\frac{1}{2} m(3-m) u(x)-\frac{1}{2} m^{2} \frac{f^{\prime \prime}(x)}{f^{\prime}(x)} u(x)^{2} \\
u(x)=\frac{f(x)}{f^{\prime}(x)} .
\end{array}
$$

See also Traub [3, (7)-(11)].

Indeed, taking $f(x)$ as

$$
f(x)=(x-a)^{m} g(x), \quad g(a) \neq 0,
$$

we have with $F_{3}(x)$ in (45) that

$$
\begin{aligned}
x_{1}-a= & \frac{1}{2 m^{2}} \frac{(3+m) g^{\prime 2}-m g g^{\prime \prime}}{g^{2}} \\
& \times\left(x_{0}-a\right)^{3}+O\left(\left(x_{0}-a\right)^{4}\right),
\end{aligned}
$$

where $g=g(a), g^{\prime}=g^{\prime}(a), g^{\prime \prime}=g^{\prime \prime}(a)$.

For more on such recursive formulas, see Traub [3, Section 8.3] and Petković et al. [7, Theorem 2 and Remark 1].

\section{A Finite-Difference Approximation}

Wishing to avoid the possibly computationally costly additional derivative in (19), we propose to approximate it by the central difference scheme

$$
\begin{aligned}
f^{\prime}\left(x_{0}+\frac{1}{2} \delta x\right) \\
=\frac{f\left(x_{0}+(1 / 2) \delta x+h\right)-f\left(x_{0}+(1 / 2) \delta x-h\right)}{2 h} \\
\quad+O\left(h^{2}\right) .
\end{aligned}
$$

Taking $h=-\delta x / 2$ leaves us with the approximation

$$
\begin{array}{r}
f^{\prime}\left(x_{0}+\frac{1}{2} \delta x\right)=\frac{f\left(x_{0}\right)-f\left(x_{0}-u_{0}\right)}{u_{0}}=(1-r) f_{0}^{\prime}, \\
\delta x=-u_{0}=-\frac{f_{0}}{f_{0}^{\prime}}, \quad r=\frac{f_{1}}{f_{0}},
\end{array}
$$

where $x_{1}=x_{0}-u_{0}, f_{1}=f\left(x_{1}\right)$, by which (19) becomes the cubic chord or two-step method

$$
x_{2}=x_{0}-\frac{1}{1-r} u_{0}, \quad u_{0}=\frac{f_{0}}{f_{0}^{\prime}},
$$

$$
x_{1}=x_{0}-u_{0}, \quad f_{1}=f\left(x_{1}\right), \quad r=\frac{f_{1}}{f_{0}} .
$$

See also Traub [3, page $180,(8)-(55)]$. We return to this method in the next section.

The second derivative approximation

$$
\begin{aligned}
& f(x-u)=f(x)-u f^{\prime}(x)+\frac{1}{2} u^{2} f^{\prime \prime}(x) \\
&=\frac{1}{2} u^{2} f^{\prime \prime}(x), \\
& f^{\prime \prime}(x)=\frac{2}{u^{2}} f_{1}, \quad u=\frac{f}{f^{\prime}}, f_{1}=f(x-u)
\end{aligned}
$$

leads to the same result.

\section{A Cubic, One-Sided, Two-Step, Secant Method}

Having computed by Newton's method

$$
\begin{gathered}
f_{0}=f\left(x_{0}\right), \quad f_{0}^{\prime}=f^{\prime}\left(x_{0}\right), \\
x_{1}=x_{0}-u_{0}, \quad u_{0}=\frac{f_{0}}{f_{0}^{\prime}}, \quad f_{1}=f\left(x_{1}\right),
\end{gathered}
$$

we propose to proceed and predict the next $x_{2}$ by pseudoNewton's method

$$
\begin{gathered}
x_{2}=x_{1}-\frac{f_{1}}{g_{1}^{\prime}}, \quad x_{1}=x_{0}-u_{0}, \\
g_{1}^{\prime}=\frac{f_{0}-f_{1}}{x_{0}-x_{1}}=(1-r) f_{0}^{\prime},
\end{gathered}
$$

skirting the computation of a new $f^{\prime}\left(x_{1}\right)$. In (53)

$$
\begin{array}{r}
r=\frac{f_{1}}{f_{0}}=\frac{f\left(x_{0}-u_{0}\right)}{f_{0}}=\frac{1}{2} \frac{f^{\prime \prime}}{f^{\prime}}\left(x_{0}-a\right)+O\left(x_{0}-a\right)^{2}, \\
f^{\prime}=f^{\prime}(a), \quad f^{\prime \prime}=f^{\prime \prime}(a) .
\end{array}
$$

We write (53) variously as

$$
\begin{gathered}
x_{2}=x_{0}-\frac{1}{1-r} u_{0} \quad \text { or } \quad x_{2}=x_{0}-(1+r) u_{0} \\
\text { or } \quad x_{2}=x_{0}-\left(1+r+r^{2}\right) u_{0}, \quad r=\frac{f_{1}}{f_{0}}
\end{gathered}
$$


with all three methods being cubic

$$
\begin{aligned}
& x_{2}-a=\frac{1}{4}\left(\frac{f^{\prime \prime}}{f^{\prime}}\right)^{2}\left(x_{0}-a\right)^{3}, \\
& x_{2}-a=\frac{1}{2}\left(\frac{f^{\prime \prime}}{f^{\prime}}\right)^{2}\left(x_{0}-a\right)^{3}, \\
& x_{2}-a=\frac{1}{4}\left(\frac{f^{\prime \prime}}{f^{\prime}}\right)^{2}\left(x_{0}-a\right)^{3} .
\end{aligned}
$$

Notice the extra $r^{2}$ in the last method of (55), added to recover the factor $1 / 4$ in the last of error equations (56).

Convergence is here one sided: if $x_{0}-a>0$, then $x_{2}-a>$ 0 ; and if $x_{0}-a<0$, then $x_{2}-a<0$.

For example, for $f(x)=x+x^{2}$, we obtain by the first method of (55) the two oppositely or contrarily converging sequences

$$
\begin{gathered}
x_{2}=\left\{-\frac{1}{6},-\frac{1}{126},-\frac{1}{1,953,126}\right\}, \\
x_{2}=\left\{\frac{1}{4}, \frac{1}{28}, \frac{1}{1,953,124}\right\}
\end{gathered}
$$

by which $\operatorname{root} a=0$ is bounded as

$$
\frac{-1}{1,953,126}<a<\frac{1}{1,953,124} .
$$

Method (55) is also obtained from the secant line

$$
\begin{gathered}
g(x)=A x+B, \quad A=\frac{f_{1}-f_{0}}{x_{1}-x_{0}}, \\
B=\frac{x_{1} f_{0}-x_{0} f_{1}}{x_{1}-x_{0}},
\end{gathered}
$$

passing through the two points, $\left(x_{0}, f_{0}\right),\left(x_{1}=x_{0}-u_{0}, f_{1}=\right.$ $f\left(x_{1}\right)$ ), and then taking the root of $g(x)=0$ as the next

$$
x_{2}=-\frac{B}{A}=\frac{x_{1}-r x_{0}}{1-r}=x_{0}-\frac{1}{1-r} u_{0}, \quad r=\frac{f_{1}}{f_{0}} .
$$

Including $f_{0}^{\prime}$ in the polynomial interpolation and passing a parabola through the available data $\left(x_{0}, f_{0}, f_{0}^{\prime}\right)$ and $\left(x_{1}, f_{1}\right)$ should allow us to obtain a better approximation for $f^{\prime}\left(x_{2}\right)$, and with it a higher-order method, as we will see next.

\section{Quartic Two-Step Methods}

Seeking a possibly higher-order method, we write the slope estimate of $(53)$ as $g_{1}^{\prime}=(1-P r) u_{0}$ for undetermined coefficient $P$ and then advantageously determine it, to have

$$
\begin{gathered}
x_{2}=x_{1}-\frac{f_{1}}{g_{1}^{\prime}}, \quad g_{1}^{\prime}=(1-2 r) f_{0}^{\prime}, \\
x_{1}=x_{0}-u_{0}, \quad f_{1}=f\left(x_{1}\right), \quad r=\frac{f_{1}}{f_{0}}
\end{gathered}
$$

or

$$
x_{2}=x_{0}-\frac{1-r}{1-2 r} u_{0}
$$

which is the celebrated quartic

$$
x_{2}-a=\frac{1}{24} \frac{f^{\prime \prime}\left(3 f^{\prime \prime 2}-2 f^{\prime} f^{\prime \prime \prime}\right)}{f^{\prime 3}}\left(x_{0}-a\right)^{4}+O\left(\left(x_{0}-a\right)^{5}\right)
$$

method of Ostrowski [8]. See also Traub [3, page 184, (8)-(78)] and King [9]. Quartic method (62) is also obtained by replacing $f_{1}$ by $2 f_{1}$ in the slope estimate $g_{1}^{\prime}$ in (53).

The method

$$
\begin{aligned}
& x_{2}=x_{1}-\frac{f_{1}}{g_{1}^{\prime}}, \quad g_{1}^{\prime}=\left(1-2 r-r^{2}\right) f_{0}^{\prime}, \\
& x_{1}=x_{0}-u_{0}, \quad f_{1}=f\left(x_{1}\right), \quad r=\frac{f_{1}}{f_{0}}
\end{aligned}
$$

is quartic as well but with the simpler error equation

$$
x_{1}-a=\frac{1}{12} \frac{f^{\prime \prime} f^{\prime \prime \prime}}{f^{\prime 2}}\left(x_{0}-a\right)^{4}+O\left(\left(x_{0}-a\right)^{5}\right) \text {. }
$$

Power series expansion changes rational method (62) into the polynomial in $r$ method

$$
x_{2}=x_{0}-\left(1+r+2 r^{2}\right) u_{0}, \quad r=\frac{f_{1}}{f_{0}}
$$

which is still quartic

$$
\begin{aligned}
x_{2}-a= & \frac{1}{24} \frac{f^{\prime \prime}}{f^{\prime 3}}\left(15 f^{\prime \prime 2}-2 f^{\prime} f^{\prime \prime \prime}\right)\left(x_{0}-a\right)^{4} \\
& +O\left(\left(x_{0}-a\right)^{5}\right) .
\end{aligned}
$$

The quartic method of (66) is also obtained from the parabola passing through the data $\left(x_{0}, f_{0}, f_{0}^{\prime}\right)$ and $\left(x_{1}, f_{1}\right)$

$$
\begin{array}{r}
g(x)=A x^{2}+B x+C, \quad A=r \frac{f_{0}^{\prime 2}}{f_{0}}, B=f_{0}^{\prime}-2 A x_{0}, \\
C=f_{0}-B x_{0}-A x_{0}^{2}
\end{array}
$$

with the predicted $x_{2}$ such that $g\left(x_{2}\right)=0$ or by taking $g_{1}^{\prime}=$ $2 A x_{1}+B$ in pseudo-Newton's method $x_{2}=x_{1}-f_{1} / g_{1}^{\prime}$.

Replacing $g_{1}^{\prime}$ in (61) by the perturbed slope

$$
g_{1}^{\prime}=(1-2 r-2 k r) f_{0}^{\prime}, \quad k>0
$$

turns the method into the supercubic alternatingly converging method

$$
x_{2}-a=-\frac{1}{2} k\left(\frac{f^{\prime \prime}}{f^{\prime}}\right)^{2}\left(x_{0}-a\right)^{3}+O\left(\left(x_{0}-a\right)^{4}\right) .
$$




\section{Quintic, Sextic, Septic, and Octic Three-Step Methods}

We continue with higher-order multistep methods requiring only the sole derivative $f_{0}^{\prime}=f^{\prime}\left(x_{0}\right)$ at initial point $x_{0}$.

The pseudo-Newton method

$$
\begin{gathered}
x_{3}=x_{2}-\frac{f_{2}}{g_{2}^{\prime}}, \quad g_{2}^{\prime}=\frac{f_{1}-f_{2}}{x_{1}-x_{2}}, \\
x_{2}=x_{0}-\frac{1}{1-r} u_{0}, \quad f_{2}=f\left(x_{2}\right)
\end{gathered}
$$

or

$$
\begin{gathered}
x_{3}=x_{2}-\frac{f_{2}}{g_{2}^{\prime}}, \quad g_{2}^{\prime}=(1-r)(1-s) f_{0}^{\prime}, \quad r=\frac{f_{1}}{f_{0}}, \\
s=\frac{f_{2}}{f_{1}}, \quad f_{1}=f\left(x_{1}\right), \quad f_{2}=f\left(x_{2}\right)
\end{gathered}
$$

is quintic and one sided

$$
x_{3}-a=\frac{1}{16}\left(\frac{f^{\prime \prime}}{f^{\prime}}\right)^{4}\left(x_{0}-a\right)^{5}+O\left(\left(x_{0}-a\right)^{6}\right) \text {. }
$$

The method

$$
\begin{gathered}
x_{3}=x_{2}-\frac{f_{2}}{g_{2}^{\prime}}, \quad x_{2}=x_{0}-\left(1+r+2 r^{2}\right) u_{0}, \\
g_{2}^{\prime}=(1-r)(1-s) f_{0}^{\prime}, \quad r=\frac{f_{1}}{f_{0}}, \quad s=\frac{f_{2}}{f_{1}}
\end{gathered}
$$

is sextic

$$
\begin{aligned}
x_{3}-a= & \frac{1}{96} \frac{f^{\prime \prime 3}\left(15 f^{\prime \prime 2}-2 f^{\prime} f^{\prime \prime \prime}\right)}{f^{\prime 5}}\left(x_{0}-a\right)^{6} \\
& +O\left(\left(x_{0}-a\right)^{7}\right) .
\end{aligned}
$$

The method

$$
\begin{gathered}
x_{3}=x_{2}-\frac{f_{2}}{g_{2}^{\prime}}, \quad x_{2}=x_{0}-\frac{1}{1-r} u_{0}, \\
g_{2}^{\prime}=\left(1-r+r^{2}-s\left(1+r-2 r^{2}\right)\right) f_{0}^{\prime}, \quad r=\frac{f_{1}}{f_{0}}, \quad s=\frac{f_{2}}{f_{1}}
\end{gathered}
$$

is septic

$$
\begin{aligned}
x_{3}-a= & \frac{1}{192} \frac{f^{\prime \prime 3}\left(9 f^{\prime \prime 3}-6 f^{\prime} f^{\prime \prime} f^{\prime \prime \prime}+f^{\prime 2} f^{(4)}\right)}{f^{\prime 6}}(x-a)^{7} \\
& +O\left(\left(x_{0}-a\right)^{8}\right) .
\end{aligned}
$$

The method

$$
\begin{gathered}
x_{3}=x_{2}-\frac{f_{2}}{g_{2}^{\prime}}, \quad x_{2}=x_{0}-\left(1+r+2 r^{2}\right) u_{0}, \\
g_{2}^{\prime}=\left(1-2 r+3 r^{2}-s\right) f_{0}^{\prime},
\end{gathered}
$$

where $r=f_{1} / f_{0}$ and $s=f_{2} / f_{1}$, is octic

$$
\begin{aligned}
x_{3}-a= & \frac{1}{1152} \\
& \times\left(f^{\prime \prime 2}\left(15 f^{\prime \prime 2}-2 f^{\prime} f^{\prime \prime \prime}\right)\right. \\
& \left.\quad \times\left(3 f^{\prime \prime 3}-6 f^{\prime} f^{\prime \prime} f^{\prime \prime \prime}+f^{\prime 2} f^{(4)}\right)\right) \\
& \times\left(f^{\prime 7}\right)^{-1}\left(x_{0}-a\right)^{8} \\
& +O\left(\left(x_{0}-a\right)^{9}\right) .
\end{aligned}
$$

See also $[7,10-16]$.

Octic method (78) is also obtained from a cubic polynomial $g(x)$ passing through the data $\left(x_{0}, f_{0}, f_{0}^{\prime}\right),\left(x_{1}, f_{1}\right)$, $\left(x_{2}, f_{2}\right)$, and $x_{3}$ chosen such that $g\left(x_{3}\right)=0$.

However, if the root repeats, that is, if $f^{\prime}(a)=0$, then the order of convergence of the method plummets from eighth order to first order.

\section{Estimates for the Root Multiplicity Index}

In this section we derive both first and second order estimates for the root multiplicity index $m$. Also derived is an estimate for the relative size of the second term in the Taylor expansion of function $f(x)$ at root point $a$.

Assuming that the power series expansion of function $f(x)$, whose root $a$ we are seeking, is of the form

$$
f(x)=(x-a)^{m}\left(A+B(x-a)+C(x-a)^{2}+\cdots\right),
$$

$$
m \geq 1
$$

we obtain from it the first order estimate for the root multiplicity index $m$

$$
\begin{gathered}
u^{\prime}=\frac{1}{m}-\frac{2}{m^{2}} \frac{B}{A}(x-a)+O\left((x-a)^{2}\right), \quad u=\frac{f}{f^{\prime}}, \\
-\frac{1}{2} \frac{u^{\prime \prime}}{u^{\prime 2}}=\frac{B}{A}+O((x-a))
\end{gathered}
$$

as well as the second order estimate for $m$

$$
\begin{aligned}
u^{\prime 2}-2 u u^{\prime \prime}= & \frac{1}{m^{2}}+\frac{6}{m^{4}} \frac{-B^{2}(1+m)+2 A C m}{A^{2}}(x-a)^{2} \\
& +O\left((x-a)^{3}\right) .
\end{aligned}
$$

For example, for $f=x^{2}+x^{3}, m=2$, we compute, at $x=0.1$, the first order and second order approximations

$$
m=2.18, \quad m=2.03
$$

respectively. For $x=0.1$ and $x=0.01$, we compute from (82)

$$
\frac{B}{A}=0.78, \quad \frac{B}{A}=0.9753,
$$

respectively. 
We have also that

$$
\begin{gathered}
\frac{f\left(x_{0}-k u_{0}\right)}{f\left(x_{0}\right)}=\left(1-\frac{k}{m}\right)^{m}+O\left(\left(x_{0}-a\right)\right), \\
\frac{f\left(x_{0}-m u_{0}\right)}{f\left(x_{0}\right)}=\frac{1}{m^{m}}\left(\frac{B}{A}\right)^{3}\left(x_{0}-a\right)^{3}+O\left(\left(x_{0}-a\right)^{4}\right) .
\end{gathered}
$$

From the first of the previous equations we have, for $k=1$,

$$
\ln r=m \ln \left(1-\frac{1}{m}\right), \quad r=\frac{f\left(x_{0}-k u_{0}\right)}{f\left(x_{0}\right)},
$$

which, with the Padé rational approximation

$$
\ln (1+x)=x \frac{x+6}{4 x+6}+R, \quad R(x \sim 0)=-\frac{1}{36} x^{4},
$$

becomes the first order estimate for the multiplicity index

$$
m=\frac{1}{6} \frac{1+4 \ln r}{1+\ln r}, \quad r=\frac{f\left(x_{0}-u_{0}\right)}{f\left(x_{0}\right)}, \quad u_{0}=\frac{f_{0}}{f_{0}^{\prime}} .
$$

For example, for $f(x)=x^{3}+x^{4}, x=\{1.0,0.5,0.1\},(89)$ yields the approximations $m=\{3.72,3.51,3.14\}$ for the exact $m=3$.

Traub [3, Section 7.8] has the pointwise approximation

$$
m=\frac{\ln |f(x)|}{\ln |u(x)|}
$$

which yields for $f(x)=x^{3}+x^{4}, x=\{0.1,0.01\}$, the less precise estimates $m=\{1.99,2.42\}$.

Suppose that $m>1$. Application of Newton's method $x_{1}=x_{0}-f_{0} / f_{0}^{\prime}$ to find a root of multiplicity greater than one reduces the method to first order

$$
\begin{aligned}
x_{1}-a= & \left(1-\frac{1}{m}\right)\left(x_{0}-a\right)+\frac{1}{m^{2}} \frac{B}{A}\left(x_{0}-a\right)^{2} \\
& +O\left(\left(x_{0}-a\right)^{3}\right) .
\end{aligned}
$$

Ignoring higher-order terms and eliminating root $a$ from the pair of successive approximations

$x_{1}-a=\left(1-\frac{1}{m}\right)\left(x_{0}-a\right), \quad x_{2}-a=\left(1-\frac{1}{m}\right)\left(x_{1}-a\right)$

leave us with the discrete, first order approximation

$$
\begin{aligned}
\mu= & \frac{x_{0}-x_{1}}{u_{0}-u_{1}}=\frac{x_{0}-x_{1}}{x_{0}-2 x_{1}+x_{2}}=m+\frac{B}{A} \frac{2 m-1}{m}\left(x_{0}-a\right) \\
& +O\left(\left(x_{0}-a\right)^{2}\right)
\end{aligned}
$$

to the multiplicity index $m$ of the root, an approximation which is the discrete counterpart to that in (81).
Now

$$
\begin{gathered}
x_{3}=\mu x_{2}-(\mu-1) x_{1} \text { or } x_{2}=x_{1}-\mu \frac{f_{1}}{f_{1}^{\prime}}, \\
\mu=\frac{x_{0}-x_{1}}{u_{0}-u_{1}}
\end{gathered}
$$

is quadratic

$$
x_{2}-a=-\frac{B}{A} \frac{m-1}{m^{2}}\left(x_{0}-a\right)^{2}+O\left(\left(x_{0}-a\right)^{3}\right)
$$

with no need for prior knowledge of $m$.

For example, using method (94), with the updated $\mu$ employed in the computation of $x_{1}$ in each cycle, we generate the alternatingly converging sequences $x_{2}=\{1.0,-6.5$. $\left.10^{-2}, 4.0 \cdot 10^{-4},-9.2 \cdot 10^{-10}\right\}$ and $m=\{3.73,2.95,3.0004\}$. Actually, for a pair of successively computed $x$ values in the sequence, $\left|x_{1}\right|=0.43\left|x_{0}\right|^{2.6}$.

For a high-order method, to realize its full speed of convergence, it is necessary that the estimated $m$ is appropriately accurate. For example, using the estimate for $m$ from (90) in the modified Newton method $x_{1}=x_{0}-m f_{0} / f_{0}^{\prime}$, we obtain for $f(x)=x^{2}+x^{3}$ the sequences $m=\{1.27,1.52,1.68,1.78,1.83\}$ and $x=\{0.2,0.082,0.022,0.0037,0.00042,0.000032\}$, with convergence, that is, barely above the linear: $x_{1}=0.32\left(x_{0}\right)^{p}$, $p=1.184$.

\section{Correction for Multiple Roots by Undetermined Coefficients}

We rewrite Newton's method as

$$
x_{1}=x-P u_{0}, \quad u_{0}=\frac{f_{0}}{f_{0}^{\prime}}
$$

for the undetermined coefficient $P$ and have that near a root of multiplicity $m \geq 1$

$$
\begin{aligned}
x_{1}-a= & \left(1-\frac{P}{m}\right)\left(x_{0}-a\right)+\frac{P}{m^{2}} \frac{B}{A}\left(x_{0}-a\right)^{2} \\
& +O\left(\left(x_{0}-a\right)^{3}\right) .
\end{aligned}
$$

Quadratic convergence is restored, as is well known, with $P=$ $m$. In the previous equation, $A$ and $B$ are the coefficients in the power series expansion of $f(x)$ in (80).

With $P=m(1-k), k<0$, the modified Newton method of (96) is reduced to an alternating superlinear method. For example, for $f(x)=x^{2}+x^{3}, m=2, k=-1 / 8$, we generate, starting with $x_{0}=1$,

$$
\begin{aligned}
x_{1}=\left\{1,1.0 \cdot 10^{-1},-7.6 \cdot 10^{-3}, 9.8 \cdot 10^{-4},\right. \\
\left.-1.2 \cdot 10^{-4}, 1.5 \cdot 10^{-5},-1.9 \cdot 10^{-6}\right\} .
\end{aligned}
$$

Next, we rewrite the method in (50) as

$$
\begin{gathered}
x_{2}=x_{0}-\frac{P}{Q-r} u_{0}, \quad r=\frac{f_{1}}{f_{0}}, f_{1}=f\left(x_{1}\right), \\
x_{1}=x_{0}-u_{0}
\end{gathered}
$$


and seek to adjust correction coefficients $P$ and $Q$ so that convergence remains cubic even to root $a$ of multiplicity $m>$ 1. By power series expansion we determine that

$$
\begin{gathered}
P=Q=\left(\frac{m-1}{m}\right)^{m-1}, \quad m>1, \\
P=Q=1 \text { if } m=1
\end{gathered}
$$

upholds cubic convergence

$$
x_{2}-a=\frac{m B^{2}-2(m-1) A C}{2 m^{2} A^{2}}\left(x_{0}-a\right)^{3}+O\left(\left(x_{0}-a\right)^{4}\right),
$$

where $A, B$, and $C$ are as in (80). Method (99)-(100) is found in [17]. See also [18].

The method

$$
\begin{gathered}
x_{2}=x_{0}-(P+Q r) u_{0}, \quad P=m(2-m), \\
Q=\frac{m^{m+1}}{(m-1)^{m-1}}, \quad m>1
\end{gathered}
$$

is similarly cubic

$$
\begin{aligned}
x_{2}-a= & \frac{(m+2) B^{2}-2(m-1) A C}{2 m^{2} A^{2}}\left(x_{0}-a\right)^{3} \\
& +O\left(\left(x_{0}-a\right)^{4}\right) .
\end{aligned}
$$

No such correction to account for multiple roots exists for the quartic two-step method of (62).

\section{Correction of Halley's Method for Multiple Roots}

We parametrize Halley's method of (22) with the undetermined coefficients $P$ and $Q$ as

$$
x_{1}=x_{0}-\frac{P f_{0}^{\prime}}{Q f_{0}^{\prime 2}-f_{0} f_{0}^{\prime \prime}} f_{0}
$$

and determine by power series expansion that for

$$
P=2, \quad Q=1+\frac{1}{m}
$$

convergence remains cubic even to a root of any multiplicity $m \geq 1$

$$
x_{1}-a=\frac{(m+1) B^{2}-2 m A C}{2 m^{2} A^{2}}\left(x_{0}-a\right)^{3}+O\left(\left(x_{0}-a\right)^{4}\right) \text {. }
$$

Method (104)-(105) is found in Hansen and Patrick [19].

Method (24) becomes here for a multiple root

$$
\begin{gathered}
x_{1}=x_{0}+\left(P+Q \frac{f_{0}^{\prime \prime}}{f_{0}^{\prime}} u_{0}\right) u_{0}, \quad P=\frac{1}{2} m(m-3), \\
Q=-\frac{1}{2} m^{2}
\end{gathered}
$$

with error equation (106).

\section{From a Cubic to a Quartic Method by Taylor's Formula}

We write the second order, $n=2$, version of the TaylorLagrange formula

$$
f(x)=f\left(x_{0}+\delta x\right)=f\left(x_{0}\right)+\delta x f^{\prime}\left(x_{0}\right)+\frac{1}{2} \delta x^{2} f^{\prime \prime}(\xi),
$$

$$
x_{0}<\xi<x_{0}+\delta x
$$

and take $f\left(x_{1}=x_{0}+\delta x\right)=0, \xi=x_{0}$ to obtain the iterative method

$$
x_{1}=x_{0}+\delta x, \quad 0=f\left(x_{0}\right)+\delta x f^{\prime}\left(x_{0}\right)+\frac{1}{2} \delta x^{2} f^{\prime \prime}\left(x_{0}\right) .
$$

We propose to approximate the solution of the quadratic increment equation

$$
f_{0}+\delta x f_{0}^{\prime}+\frac{1}{2} \delta x^{2} f_{0}^{\prime \prime}=0
$$

or, for that matter, any such higher-order algebraic equation, by the power series

$$
\delta x=\left(P+Q f_{0}+R f_{0}^{2}+S f_{0}^{3}+T f_{0}^{4}+\cdots\right) f_{0}
$$

and have upon substitution and collection

$$
\begin{aligned}
& \left(1+P f_{0}^{\prime}\right)+\left(Q f_{0}^{\prime}+\frac{1}{2} P^{2} f_{0}^{\prime \prime}\right) f_{0}+\left(R f_{0}^{\prime}+P Q f_{0}^{\prime \prime}\right) f_{0}^{2} \\
& +\left(S f_{0}^{\prime}+\frac{1}{2} Q^{2} f_{0}^{\prime \prime}+P R f_{0}^{\prime \prime}\right) f_{0}^{3}+\cdots=0
\end{aligned}
$$

from which we deduce, by annulling lower order terms, that

$$
\begin{gathered}
P=-\frac{1}{f_{0}^{\prime}}, \quad Q=-\frac{1}{2} P^{2} v_{0}, \quad R=-P Q v_{0}, \\
S=-\left(\frac{1}{2} Q^{2}+P R\right) v_{0}, \quad T=-(Q R+P S) v_{0}, \quad v_{0}=\frac{f_{0}^{\prime \prime}}{f_{0}^{\prime}}
\end{gathered}
$$

and so on.

The methods

$$
x_{1}=x_{0}+P f_{0}+Q f_{0}^{2}, \quad x_{2}=x_{0}+P f_{0}+Q f_{0}^{2}+R f_{0}^{3}
$$

or

$$
\begin{gathered}
x_{1}=x_{0}-\left(1+\frac{1}{2} w_{0}\right) u_{0}, \\
x_{2}=x_{0}-\left(1+\frac{1}{2} w_{0}+\frac{1}{2} w_{0}^{2}\right) u_{0}, \\
u=\frac{f}{f^{\prime}}, \quad v=\frac{f^{\prime \prime}}{f^{\prime}}, \quad w=u v
\end{gathered}
$$


are both cubic

$$
\begin{aligned}
& x_{1}-a=\frac{1}{6} \frac{3 f^{\prime \prime 2}-f^{\prime} f^{\prime \prime \prime}}{f^{\prime 2}}\left(x_{0}-a\right)^{3}+O\left(\left(x_{0}-a\right)^{4}\right), \\
& x_{2}-a=-\frac{1}{3} \frac{f^{\prime \prime \prime}}{f^{\prime}}\left(x-a_{0}\right)^{3}+O\left(\left(x_{0}-a\right)^{4}\right)
\end{aligned}
$$

provided that $f^{\prime}(a) \neq 0$.

As here $n=2$, we take next

$$
\xi=\frac{2}{3} x_{0}+\frac{1}{3} x_{1}
$$

recalculate $f^{\prime \prime}(\xi)$, and verify that the second method in (114) is elevated thereby to quartic

$$
x_{1}-a=\frac{1}{72}\left(\frac{45 f^{\prime \prime 3}}{f^{\prime 3}}+\frac{f^{(4)}}{f^{\prime}}\right)\left(x_{0}-a\right)^{4}+O\left(\left(x_{0}-a\right)^{5}\right) .
$$

\section{Contrarily Converging Superquadratic Methods}

We write

$$
\begin{gathered}
x_{2}=x_{0}-(1+\operatorname{Pr}) u_{0}, \quad u_{0}=\frac{f_{0}}{f_{0}^{\prime}}, \quad r=\frac{f_{1}}{f_{0}}, \\
f_{1}=f\left(x_{1}\right), \quad x_{1}=x_{0}-u_{0}
\end{gathered}
$$

for undetermined coefficient $P$ and have

$$
x_{2}-a=\frac{1}{2} \frac{f^{\prime \prime}}{f^{\prime}}(1-P)\left(x_{0}-a\right)^{2}+O\left(\left(x_{0}-a\right)^{3}\right) .
$$

We request that

$$
\frac{f^{\prime \prime}}{f^{\prime}}(1-P)=2 k\left(\frac{f^{\prime \prime}}{f^{\prime}}\right)^{2},
$$

for parameter $k$, or, in view of (51), that

$$
P=1-4 k \frac{r}{u_{0}},
$$

by which the iterative method in (119) becomes

$$
\begin{gathered}
x_{2}=x_{0}-(1+r) u_{0}+4 k r^{2}, \quad u_{0}=\frac{f_{0}}{f_{0}^{\prime}}, \\
x_{1}=x_{0}-u_{0}, \quad f_{1}=f\left(x_{1}\right), \quad r=\frac{f_{1}}{f_{0}}, \\
x_{2}-a=k\left(\frac{f^{\prime \prime}}{f^{\prime}}\right)^{2}\left(x_{0}-a\right)^{2}+O\left(\left(x_{0}-a\right)^{3}\right) .
\end{gathered}
$$

This superquadratic method converges from above if $k>0$, and from below if $k<0$.
The interest in the method

$$
\begin{gathered}
x_{2}=x_{0}-\frac{2}{1-r} u_{0}, \quad x_{1}=x_{0}-2 u_{0}, \quad f_{1}=f\left(x_{1}\right), \\
u_{0}=\frac{f_{0}}{f_{0}^{\prime}}, \quad r=\frac{f_{1}}{f_{0}}
\end{gathered}
$$

is that it ultimately converges oppositely to Newton's method

$$
x_{2}-a=-\frac{1}{2} \frac{f^{\prime \prime}}{f^{\prime}}\left(x_{0}-a\right)^{2}+O\left(\left(x_{0}-a\right)^{3}\right)
$$

as seen by comparing (125) with (17).

For example, for $f(x)=x+x^{2}$ and starting with $x_{0}=1 / 2$, we compute from Newton's method and from method (124), respectively,

$$
\begin{gathered}
x_{1}=\left\{\frac{1}{2}, \frac{1}{8}, \frac{1}{80}, \frac{1}{6,560}, \frac{1}{43,046,720}\right\}, \\
x_{2}=\left\{\frac{1}{2},-\frac{1}{10},-\frac{1}{82},-\frac{1}{6,562},-\frac{1}{43,046,722}\right\}, \\
\frac{1}{2}\left(x_{1}+x_{2}\right) \\
=\frac{1}{2}\left\{\frac{1}{1}, \frac{1}{40}, \frac{1}{3280}, \frac{1}{21,523,360}, \frac{1}{926,510,094,425,920}\right\}
\end{gathered}
$$

and root $a=0$ is bounded or bracketed thereby as

$$
\frac{-1}{43,046,722}<a<\frac{1}{43,046,720} .
$$

The average of Newton's method and the method of (124) is cubic

$$
\frac{1}{2}\left(x_{1}+x_{2}\right)-a=\frac{1}{6} \frac{f^{\prime \prime \prime}}{f^{\prime}}\left(x_{0}-a\right)^{3}+O\left(\left(x_{0}-a\right)^{4}\right) .
$$

The modified Halley method

$$
x_{1}=x_{0}+\frac{\operatorname{det}\left[\begin{array}{cc}
-1 & f_{0} \\
k & 2 f_{0}^{\prime}
\end{array}\right]}{\operatorname{det}\left[\begin{array}{cc}
f_{0}^{\prime} & f_{0} \\
f_{0}^{\prime \prime} & 2 f_{0}^{\prime}
\end{array}\right]} f_{0}=x_{0}-\frac{2 f_{0}^{\prime}+k f_{0}}{2 f_{0}^{\prime 2}-f_{0} f_{0}^{\prime \prime}} f_{0}
$$

is also superquadratic and one sided

$$
\begin{aligned}
x_{1}-a= & -\frac{1}{2} k\left(x_{0}-a\right)^{2}+\frac{1}{12} \frac{3 f^{\prime \prime 2}-2 f^{\prime} f^{\prime \prime \prime}+3 k f^{\prime} f^{\prime \prime}}{f^{\prime 2}} \\
& \times\left(x_{0}-a\right)^{3}+O\left(\left(x_{0}-a\right)^{4}\right) .
\end{aligned}
$$

According to error equation (130), if $k<0$, then convergence is at least asymptotically from above; if $k>0$, convergence is from below. 


\section{Alternating Superlinear and Supercubic Methods}

We start by modifying Newton's method as

$$
x_{1}=x_{0}-(1+k) \frac{f_{0}}{f_{0}^{\prime}}
$$

to have the superlinear method

$$
x_{1}-a=-k\left(x_{0}-a\right)+O\left(\left(x_{0}-a\right)^{2}\right)
$$

that ultimately converges alternatingly if $0<k<1$.

For example, for $f(x)=x+x^{2}, k=1 / 8$, and $x_{0}=1$, we compute by method (131) the alternating sequence

$$
\begin{aligned}
x_{1}=\{ & 1,2.5 \cdot 10^{-1}, 1.6 \cdot 10^{-2},-1.7 \cdot 10^{-3}, 2.1 \cdot 10^{-4}, \\
& \left.-2.7 \cdot 10^{-5}\right\}
\end{aligned}
$$

allowing us to bracket root $a=0$ as

$$
-2.6706 \cdot 10^{-5}<a<2.1406 \cdot 10^{-4}
$$

For a higher-order method, we start with the originally quartic

$$
\begin{gathered}
x_{2}=x_{0}-\left(1+r+Q r^{2}\right) u_{0}, \quad u_{0}=\frac{f_{0}}{f_{0}^{\prime}}, \\
r=\frac{f_{1}}{f_{0}}, \quad f_{1}=f\left(x_{0}-u_{0}\right)
\end{gathered}
$$

of (66) and have that

$$
\begin{array}{r}
x_{2}-a=-k\left(\frac{f^{\prime \prime}}{f^{\prime}}\right)^{2}\left(x_{0}-a\right)^{3}+O\left(\left(x_{0}-a\right)^{4}\right), \\
k=\frac{1}{4}(Q-2) .
\end{array}
$$

This supercubic method converges alternatingly if parameter $k>0$.

For example, for $f(x)=x+x^{2}$, we generate by methods (135)-(136), with $k=1$, the alternating sequence

$$
x_{2}=\left\{1,-0.012,8.34 \cdot 10^{-6},-2.32 \cdot 10^{-15}\right\}
$$

and $\operatorname{root} a=0$ is bracketed as

$$
-2.32 \cdot 10^{-15}<a<8.34 \cdot 10^{-6} \text {. }
$$

\section{Still Higher-Order Taylor Methods}

Starting with

$$
\begin{array}{r}
f(x)=f\left(x_{0}+\delta x\right)=f_{0}+\delta x f_{0}^{\prime}+\frac{1}{2} \delta x^{2} f_{0}^{\prime \prime}+\frac{1}{6} \delta x^{3} f^{\prime \prime \prime}(\xi), \\
x_{0}<\xi<x_{0}+\delta x,
\end{array}
$$

we obtain the iterative method

$$
x_{1}=x_{0}+\delta x, \quad f_{0}+\delta x f_{0}^{\prime}+\frac{1}{2} \delta x^{2} f_{0}^{\prime \prime}+\frac{1}{6} \delta x^{3} f_{0}^{\prime \prime \prime}=0
$$

where

$$
\delta x=\left(P+Q f_{0}+R f_{0}^{2}+S f_{0}^{3}+\cdots\right) f_{0}
$$

with

$$
\begin{gathered}
P=-\frac{1}{f^{\prime}}, \quad Q=\frac{1}{2} P^{3} f^{\prime \prime}, \quad R=P^{2}\left(Q f^{\prime \prime}+\frac{1}{6} P^{2} f^{\prime \prime \prime}\right), \\
S=P\left(\frac{1}{2} Q^{2} f^{\prime \prime}+P R f^{\prime \prime}+\frac{1}{2} P^{2} Q f^{\prime \prime \prime}\right) .
\end{gathered}
$$

The methods

$$
\begin{gathered}
x_{1}=x_{0}+\left(P+Q f_{0}+R f_{0}^{2}\right) f_{0}, \\
x_{2}=x_{0}+\left(P+Q f_{0}+R f_{0}^{2}+S f_{0}^{3}\right) f_{0}
\end{gathered}
$$

are both quartic

$$
x_{2}-a=\frac{1}{24} \frac{f^{(4)}}{f^{\prime}}\left(x_{0}-a\right)^{4}+O\left(\left(x_{0}-a\right)^{5}\right)
$$

provided that $f^{\prime}(a) \neq 0$.

Recalculating $f^{\prime \prime \prime}(\xi)$ at

$$
\xi=\frac{3}{4} x_{0}+\frac{1}{4} x_{1}
$$

elevates the method to quintic

$$
\begin{aligned}
x_{2}-a=\frac{1}{960 f^{\prime 4}}( & 840 f^{\prime \prime 4}-840 f^{\prime} f^{\prime \prime} f^{\prime \prime \prime}+80 f^{\prime} f^{\prime \prime \prime} \\
& \left.-3 f f^{\prime 3} f^{(5)}\right)\left(x_{0}-a\right)^{5}+O\left(\left(x_{0}-a\right)^{6}\right) .
\end{aligned}
$$

\section{Repeated Fourth-Order Method}

The repeated Newton method

$$
x_{1}=x_{0}-\frac{f_{0}}{f_{0}^{\prime}}, \quad x_{2}=x_{1}-\frac{f_{1}}{f_{1}^{\prime}}
$$

is also quartic

$$
x_{2}-a=\frac{1}{8}\left(\frac{f^{\prime \prime}}{f^{\prime}}\right)^{3}\left(x_{0}-a\right)^{4}+O\left(\left(x_{0}-a\right)^{5}\right) .
$$

Similarly, the repeated modified Newton method

$$
x_{1}=x_{0}-m \frac{f_{0}}{f_{0}^{\prime}}, \quad x_{2}=x_{1}-m \frac{f_{1}}{f_{1}^{\prime}}
$$


remains quartic even near a root of any multiplicity $m \geq 1$

$$
x_{2}-a=\frac{1}{m^{3}}\left(\frac{B}{A}\right)^{3}\left(x_{0}-a\right)^{4}+O\left(\left(x_{0}-a\right)^{5}\right) \text {. }
$$

The repeated-step method

$$
x_{1}=x_{0}-\frac{f_{0}^{\prime}}{f_{0}^{\prime 2}-f_{0} f_{0}^{\prime \prime}} f_{0}, \quad x_{2}=x_{1}-\frac{f_{1}^{\prime}}{f_{1}^{\prime 2}-f_{1} f_{1}^{\prime \prime}} f_{1}
$$

not requiring prior knowledge of the multiplicity index $m$ of the root is also quartic

$$
x_{2}-a=-\frac{1}{m^{3}}\left(\frac{B}{A}\right)^{3}\left(x_{0}-a\right)^{4}+O\left(\left(x_{0}-a\right)^{3}\right) \text {. }
$$

The two single-step methods

$$
x_{1}=x_{0}-m \frac{f_{0}}{f_{0}^{\prime}}, \quad x_{2}=x_{0}-\frac{f_{0}^{\prime}}{f_{0}^{\prime 2}-f_{0} f_{0}^{\prime \prime}} f_{0}
$$

converge contrarily

$$
\begin{aligned}
& x_{1}-a=\frac{1}{m} \frac{B}{A}\left(x_{0}-a\right)^{2}+O\left(\left(x_{0}-a\right)^{3}\right), \\
& x_{2}-a=-\frac{1}{m} \frac{B}{A}\left(x_{0}-a\right)^{2}+O\left(\left(x_{0}-a\right)^{3}\right) .
\end{aligned}
$$

Their average is a cubic method

$$
\begin{array}{r}
x_{3}-a=\frac{B^{2}(m-1)-2 A C m}{2 A^{2} m^{2}}\left(x_{0}-a\right)^{3}+O\left(\left(x_{0}-a\right)^{4}\right), \\
x_{3}=\frac{1}{2}\left(x_{1}+x_{2}\right) .
\end{array}
$$

For instance, for $f(x)=x^{2}+x^{3}+x^{4}, m=2$, we compute by the two methods in (153) the sequences

$$
\begin{aligned}
x_{1} & =\left\{0.5,1.1 \cdot 10^{-1}, 6.3 \cdot 10^{-3}, 2.0 \cdot 10^{-5}\right\}, \\
x_{2} & =\left\{0.5,-1.3 \cdot 10^{-1},-5.4 \cdot 10^{-3},-1.4 \cdot 10^{-5}\right\} \\
x_{3} & =\frac{1}{2}\left(x_{1}+x_{2}\right) \\
& =\left\{0.5,-1.9 \cdot 10^{-2}, 9.2 \cdot 10^{-4}, 5.6 \cdot 10^{-6}\right\} .
\end{aligned}
$$

\section{Stacked Higher-Order Methods and Simple Root}

Higher-order, single-step methods can be written as a builtup power series of $u=f / f^{\prime}$

$$
x_{1}=x_{0}-K_{2} u_{0} \quad \text { 2nd order }
$$

$$
\begin{gathered}
x_{1}=x_{0}-K_{2} u_{0}-K_{3} u_{0}^{2} \quad 3 \text { rd order } \\
x_{1}=x_{0}-K_{2} u_{0}-K_{3} u_{0}^{2}-K_{4} u_{0}^{3} \quad 4 \text { th order } \\
x_{1}=x_{0}-K_{2} u_{0}-K_{3} u_{0}^{2}-K_{4} u_{0}^{3}-K_{5} u_{0}^{4} \quad 5 \text { th order } \\
x_{1}=x_{0}-K_{2} u_{0}-K_{3} u_{0}^{2}-K_{4} u_{0}^{3}-K_{5} u_{0}^{4}-K_{6} u^{5}
\end{gathered}
$$

6 th order,

where

$$
\begin{gathered}
K_{2}=1, \quad K_{3}=\frac{1}{2} \frac{f^{\prime \prime}}{f^{\prime}}, \quad K_{4}=\frac{1}{6}\left(3 \frac{f^{\prime \prime 2}}{f^{\prime 2}}-\frac{f^{\prime \prime \prime}}{f^{\prime}}\right), \\
K_{5}=\frac{1}{24}\left(15 \frac{f^{\prime \prime 3}}{f^{\prime 3}}-10 \frac{f^{\prime \prime} f^{\prime \prime \prime}}{f^{\prime 2}}+\frac{f^{(4)}}{f^{\prime}}\right), \\
K_{6}=\frac{1}{120}\left(105 \frac{f^{\prime \prime 4}}{f^{\prime 4}}-105 \frac{f^{\prime \prime 2} f^{\prime \prime \prime}}{f^{\prime 3}}+10 \frac{f^{\prime \prime \prime 2}}{f^{\prime 2}}\right. \\
\left.+15 \frac{f^{\prime \prime} f^{(4)}}{f^{\prime 2}}-\frac{f^{(5)}}{f^{\prime}}\right),
\end{gathered}
$$

and so on. All evaluations in (158) are at $x=x_{0}$.

Alternatively, the method may be written as a product, such as the built-up sextic method

$$
x_{1}=x_{0}-\left(1+P_{3} u_{0}\right)\left(1+P_{4} u_{0}^{2}\right)\left(1+P_{5} u_{0}^{3}\right)\left(1+P_{6} u^{4}\right) u_{0}
$$

where

$$
\begin{gathered}
P_{3}=K_{3}, \quad P_{4}=K_{4}, \\
P_{5}=\frac{1}{24}\left(9 \frac{f^{\prime \prime \prime}}{f^{\prime 3}}-8 \frac{f^{\prime \prime} f^{\prime \prime \prime}}{f^{\prime 2}}+\frac{f^{(4)}}{f^{\prime}}\right), \\
P_{6}=\frac{1}{240}\left(165 \frac{f^{\prime \prime 4}}{f^{\prime 4}}-170 \frac{f^{\prime \prime 2} f^{\prime \prime \prime}}{f^{\prime 3}}+20 \frac{f^{\prime \prime \prime 2}}{f^{\prime 2}}\right. \\
\left.+25 \frac{f^{\prime \prime} f^{(4)}}{f^{\prime 2}}-2 \frac{f^{(5)}}{f^{\prime}}\right),
\end{gathered}
$$

with all evaluations in (160) done at $x=x_{0}$.

\section{Higher-Order Methods and Multiple Root}

The method

$$
\begin{gathered}
x_{1}=x_{0}-P u_{0}-Q u_{0}^{2}-R u_{0}^{3}, \quad u_{0}=\frac{f_{0}}{f_{0}^{\prime}}, \quad P=1, \\
Q=\frac{1}{2} \frac{f_{0}^{\prime \prime}}{f_{0}^{\prime}}, \quad R=\frac{1}{2}(k+1)\left(\frac{f_{0}^{\prime \prime}}{f_{0}^{\prime}}\right)^{2}-\frac{1}{6} \frac{f_{0}^{\prime \prime \prime}}{f_{0}^{\prime}}
\end{gathered}
$$


is supercubic

$$
x_{1}-a=-\frac{1}{2} k\left(\frac{f^{\prime \prime}}{f^{\prime}}\right)^{2}\left(x_{0}-a\right)^{3}+O\left(\left(x_{0}-a\right)^{4}\right)
$$

and converges alternatingly to a single root if $k>0$.

Similarly, the method

$$
\begin{array}{r}
x_{1}=x_{0}-P u_{0}-Q \frac{f_{0}^{\prime \prime}}{f_{0}^{\prime}} u_{0}^{2}-\left(R\left(\frac{f_{0}^{\prime \prime}}{f_{0}^{\prime}}\right)^{2}+S \frac{f_{0}^{\prime \prime \prime}}{f_{0}^{\prime}}\right) u_{0}^{3}, \\
u_{0}=\frac{f_{0}}{f_{0}^{\prime}}
\end{array}
$$

with

$$
\begin{gathered}
P=\frac{1}{6} m\left(11-6 m+m^{2}+3 k(1-m)^{2}\right), \\
Q=\frac{1}{2} m^{2}(2-m+2 k(1-m)), \\
R=\frac{1}{2} m^{3}(1+k), \quad S=-\frac{1}{6} m^{3}
\end{gathered}
$$

converges supercubically, and alternatingly if $k>0$

$$
x_{1}-a=-k \frac{2}{m^{2}}\left(\frac{B}{A}\right)^{2}\left(x_{0}-a\right)^{3}+O\left(\left(x_{0}-a\right)^{4}\right)
$$

to a root of any multiplicity $m \geq 1$.

Osada [20] suggests the concise cubic method, compared with method (107),

$$
\begin{gathered}
x_{1}=x_{0}-\frac{1}{2} m(m+1) \frac{f_{0}}{f_{0}^{\prime}}+\frac{1}{2}(m-1)^{2} \frac{f_{0}^{\prime}}{f_{0}^{\prime \prime}}, \quad m>1 \\
x_{1}-a=\frac{B^{2}(m+1)^{2}-2 A C m(m-1)}{2 A^{2} m^{2}(m-1)}\left(x_{0}-a\right)^{3} \\
+O\left(\left(x_{0}-a\right)^{4}\right),
\end{gathered}
$$

$$
=x_{0}-\frac{6 f_{0}^{\prime 2}-3 f_{0} f_{0}^{\prime \prime}}{6 f_{0}^{\prime 3}-6 f_{0} f_{0}^{\prime} f_{0}^{\prime \prime}+f_{0}^{2} f_{0}^{\prime \prime \prime}} f_{0} \text {. }
$$

is quartic if $f^{\prime}(a) \neq 0$.

The method

$$
\begin{gathered}
x_{1}=x_{0}-\frac{P f_{0}^{\prime 2}+Q f_{0} f_{0}^{\prime \prime}}{R f_{0}^{\prime 3}+S f_{0} f_{0}^{\prime} f_{0}^{\prime \prime}+f_{0}^{2} f_{0}^{\prime \prime \prime}} f_{0}, \quad P=3+\frac{3}{m}, \\
Q=-3, \quad R=2+\frac{3}{m}+\frac{1}{m^{2}}, \quad S=-P
\end{gathered}
$$

is quartic as well

$$
\begin{aligned}
x_{1}-a= & \left(g^{\prime 3}\left(1+3 m+2 m^{2}\right)+g g^{\prime} g^{\prime \prime}\left(-3 m-3 m^{2}\right)\right. \\
& \left.+g^{2} g^{\prime \prime \prime} m^{2}\right)\left(6 g^{3} m^{2}\right)^{-1}\left(x_{0}-a\right)^{4}+O\left(\left(x_{0}-a\right)^{5}\right)
\end{aligned}
$$

even in the event that root $a$ is of multiplicity $m \geq 1$. In (169), $g=g(a)$ is as in (46). See also [7].

Householder's [22] concise representation of the singlepoint iterative method of order $p+2$

$$
x_{1}=x_{0}+(p+1) \frac{\left(f^{-1}\right)_{0}^{(p)}}{\left(f^{-1}\right)_{0}^{(p+1)}}
$$

unfolds, for $p=2$, into the quartic method of (167).

An inexact value for the root multiplicity index plunges method (168) to mere first order. Indeed, replacing $m$ in (168) by $m(1+k)$ results in

$$
x_{1}-a=-\frac{k}{3+2 k}\left(x_{0}-a\right)+O\left(\left(x_{0}-a\right)^{2}\right) .
$$

To have a cubic method that does not require prior knowledge of the root multiplicity, we replace $f$ by $u$ to have

$$
x_{1}=x_{0}-v_{0}-\frac{1}{2} \frac{u_{0}^{\prime \prime}}{u_{0}^{\prime}} v_{0}^{2}, \quad v=\frac{u}{u^{\prime}}, u=\frac{f}{f^{\prime}}
$$

for which

$$
x_{1}-a=\frac{(1-m) B^{2}+2 m A C}{A^{2} m^{2}}\left(x_{0}-a\right)^{3}+O\left(\left(x_{0}-a\right)^{4}\right) .
$$

Still higher-order methods are readily thus generated.

\section{Numerical Differentiation}

All derivatives may, of course, be approximated by sufficiently accurate finite differences. Replacing $f^{\prime}$ and $f^{\prime \prime}$ in Halley's method by the central finite-difference approximations

$$
\begin{aligned}
\frac{f(x+h)-f(x-h)}{2 h}= & f^{\prime}(x)+\frac{1}{6} h^{2} f^{\prime \prime \prime}(x) \\
& +O\left(h^{4}\right), \\
\frac{f(x-h)-2 f(x)+f(x+h)}{h^{2}}= & f^{\prime \prime}(x)+\frac{1}{12} h^{2} f^{(4)}(x) \\
& +O\left(h^{4}\right)
\end{aligned}
$$

we have, after some simplification,

$$
\begin{aligned}
x_{1}-a= & h^{2}\left(\frac{1}{6} \frac{f^{\prime \prime \prime}}{f^{\prime}}\left(x_{0}-a\right)+\frac{1}{8} \frac{f^{(4)}}{f^{\prime}}\left(x_{0}-a\right)^{2}\right) \\
& +\frac{1}{12} \frac{3 f^{\prime \prime}-2 f^{\prime} f^{\prime \prime \prime}}{f^{\prime 2}}\left(x_{0}-a\right)^{3}+O\left(\left(x_{0}-a\right)^{4}\right) .
\end{aligned}
$$


Following Steffensen's suggestion (see $[23,24]$ ), we take $h=f\left(x_{0}\right)$ and have

$$
\begin{aligned}
x_{1}-a= & \frac{1}{12} \frac{3 f^{\prime \prime 2}-2 f^{\prime} f^{\prime \prime \prime}+2 f^{\prime 3} f^{\prime \prime \prime}}{f^{\prime 2}}\left(x_{0}-a\right)^{3} \\
& +O\left(\left(x_{0}-a\right)^{4}\right) .
\end{aligned}
$$

\section{Pell's Equation}

We consider now the very special case of the iterative solution of $f(x)=x^{2}-N$ for accurate rational approximations $p / q$ to $\sqrt{N}$. A pair of natural numbers $p, q$ that satisfies the general integer Pell equation

$$
p^{2}-N q^{2}=k
$$

is such that

$$
\left(\frac{p}{q}\right)^{2}=N+\frac{k}{q^{2}} \quad \text { or } \quad \frac{p}{q}=\sqrt{N}\left(1+\frac{k}{2 N q^{2}}\right)
$$

nearly, if $k /\left(N q^{2}\right) \ll 1$.

For $x_{0}=p_{0} / q_{0}$ and $x_{1}=p_{1} / q_{1}$, Newton's method $x_{1}=$ $x_{0}-f_{0} / f_{0}^{\prime}$ assumes the split form

$$
p_{1}=p_{0}^{2}+N q_{0}^{2}, \quad q_{1}=2 p_{0} q_{0}
$$

and we verify that

$$
p_{1}^{2}-N q_{1}^{2}=\left(p_{0}^{2}-N q_{0}^{2}\right)^{2}=k^{2} \quad \text { if } p_{0}^{2}-N q_{0}^{2}=k .
$$

Similarly, for Halley's method

$$
p_{1}=\left(3 N q_{0}^{2}+p_{0}^{2}\right) p_{0}, \quad q_{1}=N q_{0}^{3}+3 p_{0}^{2} q_{0}
$$

we verify that

$$
p_{1}^{2}-N q_{1}^{2}=\left(p_{0}^{2}-N q_{0}^{2}\right)^{3}=k^{3} \text { if } p_{0}^{2}-N q_{0}^{2}=k .
$$

The iterative method

$$
p_{1}=2 N p_{0} q_{0}, \quad q_{1}=p_{0}^{2}+N q_{0}^{2}
$$

written out from (124) converges quadratically from below to $\sqrt{N}$. Here

$$
p_{1}^{2}-N q_{1}^{2}=-N k^{2} \quad \text { if } p_{0}^{2}-N q_{0}^{2}=k .
$$

Still higher-order methods may be directly obtained from the expansion of

$$
p_{1}+\sqrt{N} q_{1}=\left(p_{0}+\sqrt{N} q_{0}\right)^{n}
$$

where $n=2$ is taken for Newton's method, $n=3$ is taken for Halley's method, and so on. See also $[25,26]$.

For example, for $n=4$ and $p_{0}=3, q_{0}=2, N=2$, and $p_{0}^{2}-N q_{0}^{2}=1$, we obtain from (185)

$$
p_{1}+\sqrt{N} q_{1}=(3+\sqrt{N} 2)^{4}=577+\sqrt{N} 408,
$$

and $p_{1}=577, q_{1}=408, p_{1}^{2}-N q_{1}^{2}=1$, and $\left(p_{1} / q_{1}\right)^{2}=2.000006$.

\section{Ratio and Mediant Corrections}

In this simplest of all iterative methods, we start with $p / q$ and add 1 to the numerator $p$ if the ratio is an underestimate, or add 1 to the denominator $q$ if the ratio is an overestimate. For example, seeking accurate rational approximations to the root of $f(x)=x^{3}-2$, we start with $p / q=4 / 4$, carry out 625 such corrections, and secure the bounds

$$
\frac{286}{227}<\sqrt[3]{2}<\frac{349}{277}, \quad \frac{349}{277}-\frac{286}{227}=\frac{1}{62,879} .
$$

The mediant fraction $M$ of the upper and lower bounds in (187) is

$$
M=\frac{286+349}{227+277}=\frac{635}{504} .
$$

We verify that $M^{3}<2$ and have the closer bounds

$$
\frac{635}{504}<\sqrt[3]{2}<\frac{349}{277}, \quad \frac{349}{277}-\frac{635}{504}=\frac{1}{139,608}
$$

We recall this well-known approximation theorem.

Theorem 2. Let $k=m / n$ be a positive rational number in lowest terms. For any number $\epsilon>0$ there is only a finite number of rational numbers, $p / q \neq m / n$, such that

$$
\left|k-\frac{p}{q}\right|<\frac{1}{q^{1+\epsilon}}, \quad \epsilon>0 .
$$

Proof. Since $m / n-p / q \neq 0$, the absolute value inequality may be recast as

$$
1 \leq|m q-n p|<\frac{n}{q^{\epsilon}}, \quad \frac{n}{q^{\epsilon}} \geq 1, \quad \epsilon>0 .
$$

Only a limited number of integers $q$ satisfy the inequality $n>$ $q^{\epsilon}$. Consequently, there is only a limited number of integers $p$ that satisfy the absolute value inequality of (191). End of proof.

Unlike the classical method of bisections that may continue indefinitely, the method of mediants that generates rational approximations as in (190) terminates in a finite number of steps if the number it seeks to trap is rational. Conversely, if the method of mediants does not terminate in a finite number of steps, then the number being trapped is surely irrational.

\section{Conflict of Interests}

The author hereby declares that there is no conflict of interests regarding the publication of this paper.

\section{References}

[1] P. R. Beesack, "A general form of the remainder in Taylor's theorem," The American Mathematical Monthly, vol. 73, no. 1, pp. 64-67, 1966. 
[2] E. I. Poffald, “The remainder in Taylor's formula," The American Mathematical Monthly, vol. 97, no. 3, pp. 205-213, 1990.

[3] J. F. Traub, Iterative Methods for the Solution of Equations, Chelsea Publishing Company, New York, NY, USA, 1977.

[4] C. Chun and B. Neta, "Some modification of Newton's method by the method of undetermined coefficients," Computers \& Mathematics with Applications, vol. 56, no. 10, pp. 2528-2538, 2008.

[5] R. Behl, V. Kanwar, and K. K. Sharma, "Another simple way of deriving several iterative functions to solve nonlinear equations," Journal of Applied Mathematics, vol. 2012, Article ID 294086, 22 pages, 2012.

[6] I. Fried, "High-order iterative bracketing methods," International Journal for Numerical Methods in Engineering, vol. 94, no. 7, pp. 708-714, 2013.

[7] M. S. Petković, L. D. Petković, and J. Džunić, "Accelerating generators of iterative methods for finding multiple roots of nonlinear equations," Computers \& Mathematics with Applications, vol. 59, no. 8, pp. 2784-2793, 2010.

[8] A. Ostrowski, Solution of Equations and Systems of Equations, Academic Press, New York, NY, USA, 1966.

[9] R. F. King, "A family of fourth order methods for nonlinear equations," SIAM Journal on Numerical Analysis, vol. 10, pp. 876-879, 1973.

[10] M. S. Petković, "On optimal multipoint methods for solving nonlinear equations," Novi Sad Journal of Mathematics, vol. 39, no. 1, pp. 123-130, 2009.

[11] B. Neta, "A sixth-order family of methods for nonlinear equations," International Journal of Computer Mathematics B, vol. 7, no. 2, pp. 157-161, 1979.

[12] D. B. Popovski, "A note on Neta's family of sixth-order methods for solving equations," International Journal of Computer Mathematics, vol. 10, no. 1, pp. 91-93, 1981.

[13] S. K. Khattri and I. K. Argyros, "How to develop fourth and seventh order iterative methods?" Novi Sad Journal of Mathematics, vol. 40, no. 2, pp. 61-67, 2010.

[14] W. Bi, H. Ren, and Q. Wu, "Three-step iterative methods with eighth-order convergence for solving nonlinear equations," Journal of Computational and Applied Mathematics, vol. 225, no. 1, pp. 105-112, 2009.

[15] R. Thukral, "A family of three-point methods of eighth-order for finding multiple roots of nonlinear Equations," Journal of Modern Methods in Numerical Mathematics, vol. 4, no. 2, pp. $1-9,2013$.

[16] R. Thukral and M. S. Petković, "A family of three-point methods of optimal order for solving nonlinear equations," Journal of Computational and Applied Mathematics, vol. 233, no. 9, pp. 2278-2284, 2010.

[17] D. Chen, "A basic theorem on constructing an iterative formula of higher order for computing multiple roots of an equation," Mathematica Numerica Sinica, vol. 4, no. 4, pp. 445-450, 1982.

[18] H. D. Victory, Jr. and B. Neta, "A higher order method for multiple zeros of nonlinear functions," International Journal of Computer Mathematics, vol. 12, no. 3-4, pp. 329-335, 1982/83.

[19] E. Hansen and M. Patrick, "A family of root finding methods," Numerische Mathematik, vol. 27, no. 3, pp. 257-269, 1976/77.

[20] N. Osada, "An optimal multiple root-finding method of order three," Journal of Computational and Applied Mathematics, vol. 51, no. 1, pp. 131-133, 1994.

[21] C. Chun, H. J. Bae, and B. Neta, "New families of nonlinear third-order solvers for finding multiple roots," Computers \&
Mathematics with Applications, vol. 57, no. 9, pp. 1574-1582, 2009.

[22] A. S. Householder, The Numerical Treatment of a Single Nonlinear Equation, McGraw-Hill, New York, NY, USA, 1970.

[23] I. F. Steffensen, "Remarks on iteration," Scandinavian Actuarial Journal, vol. 16, no. 1, pp. 64-72, 1933.

[24] X. Y. Wu, J. L. Xia, and R. Shao, "Quadratically convergent multiple roots finding method without derivatives," Computers \& Mathematics with Applications, vol. 42, no. 1-2, pp. 115-119, 2001.

[25] E. J. Barbeau, Pell's Equation, Springer, New York, NY, USA, 2000.

[26] A. McBride, "Remarks on Pell's equation and square root algorithms," The Mathematical Gazette, vol. 83, pp. 47-52, 1999. 


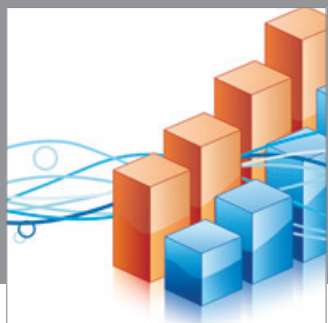

Advances in

Operations Research

mansans

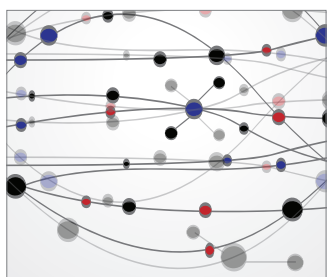

The Scientific World Journal
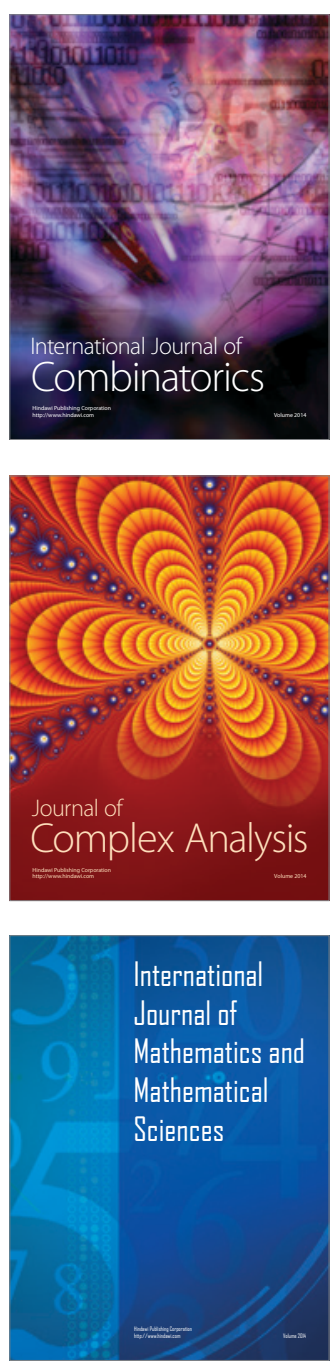
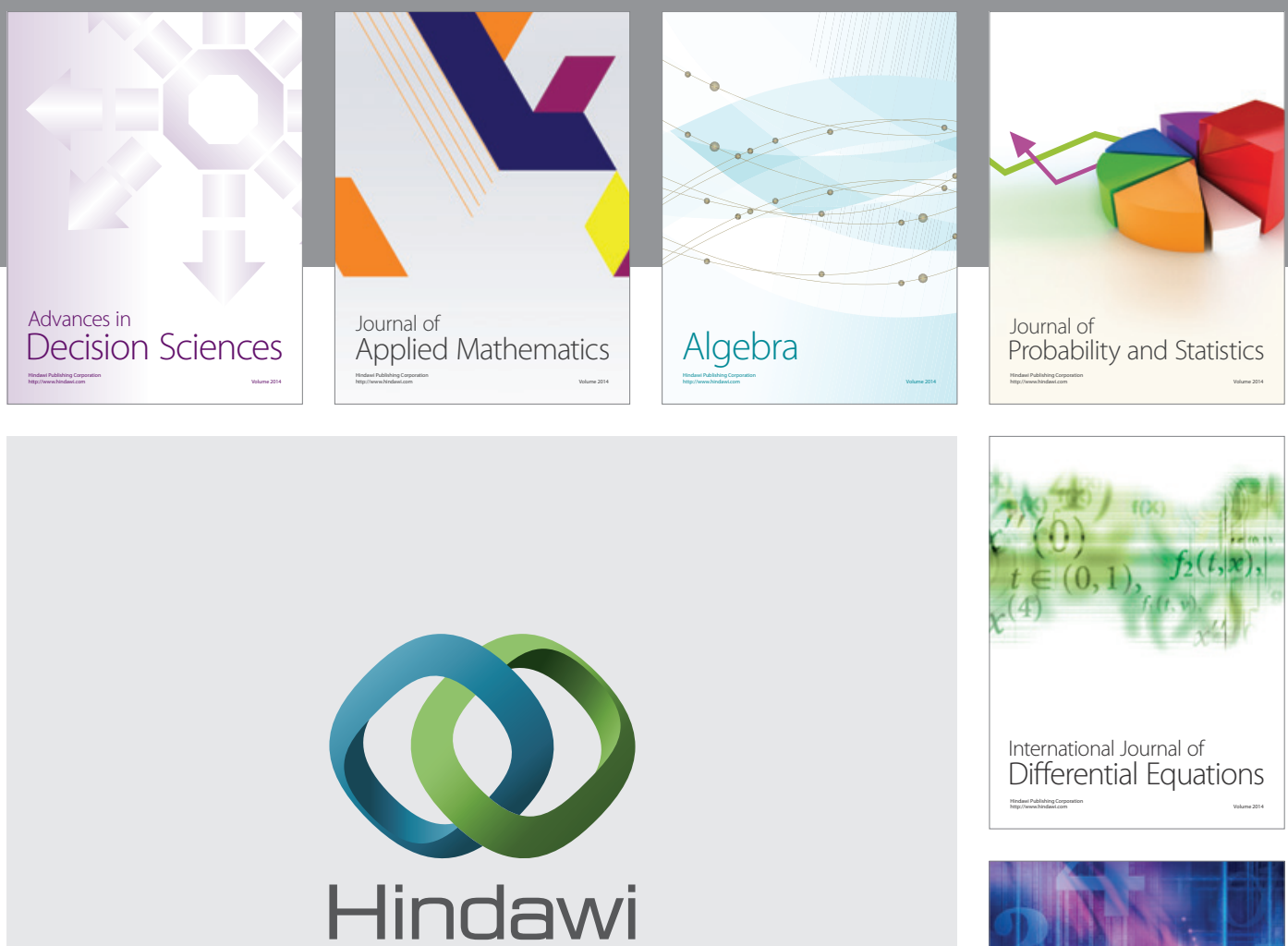

Submit your manuscripts at http://www.hindawi.com
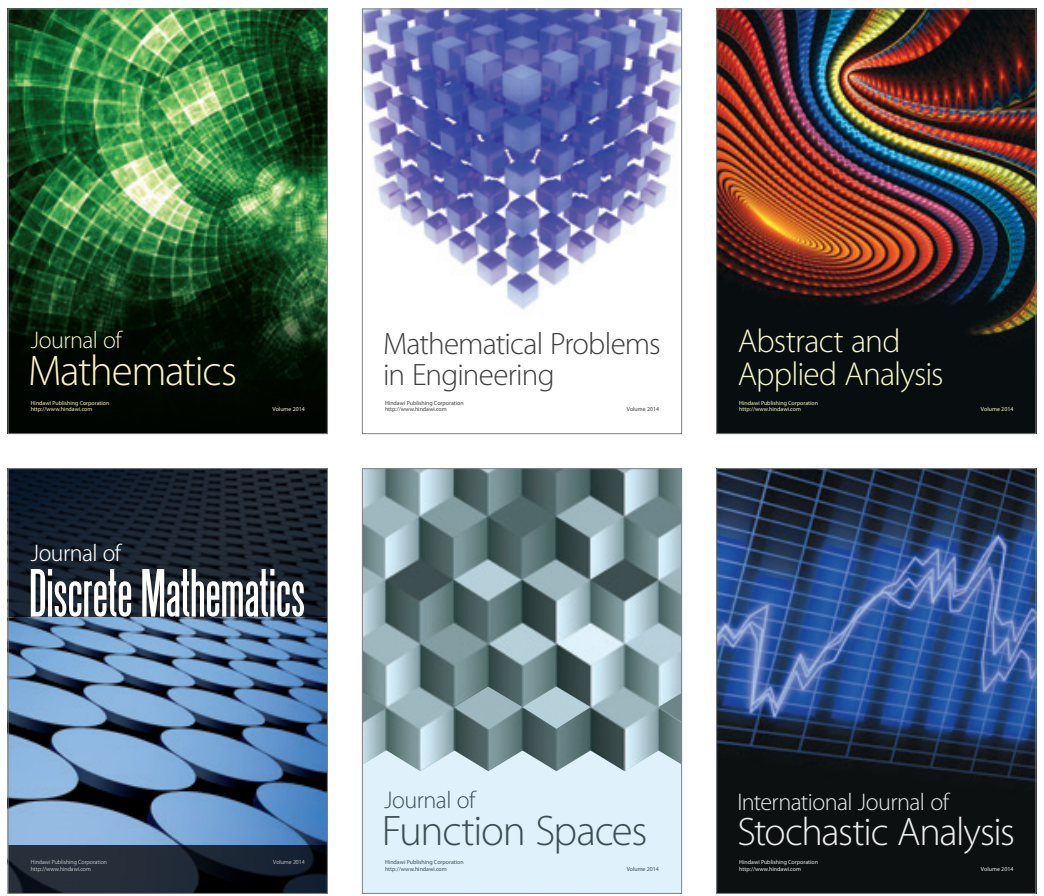

Journal of

Function Spaces

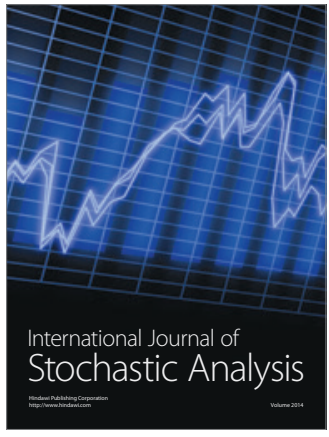

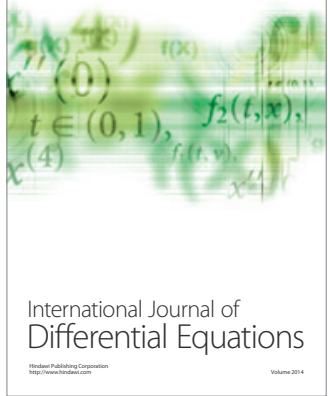
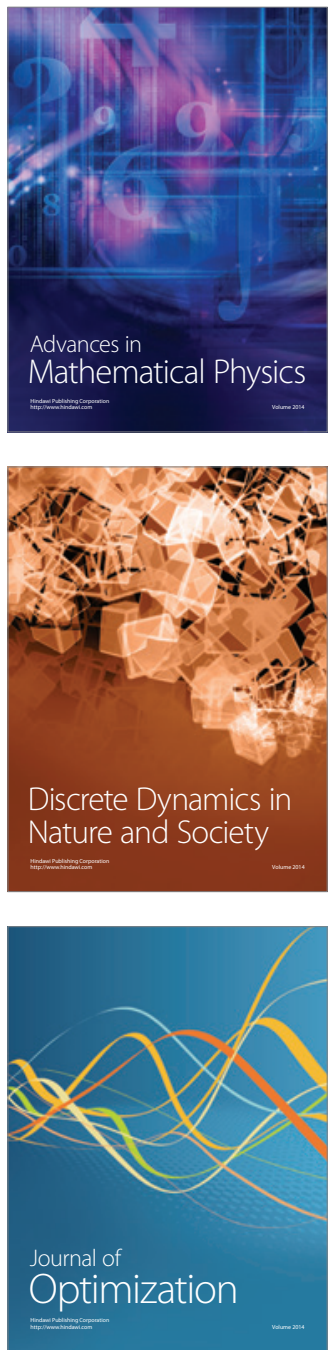